\title{
Glutamate Decreases Mitochondrial Size and Movement in Primary Forebrain Neurons
}

\author{
Gordon L. Rintoul, Anthony J. Filiano, Jacques B. Brocard, Geraldine J. Kress, and Ian J. Reynolds \\ Department of Pharmacology, University of Pittsburgh, Pittsburgh, Pennsylvania 15261
}

\begin{abstract}
Mitochondria are essential to maintain neuronal viability. In addition to the generation of ATP and maintenance of calcium homeostasis, the effective delivery of mitochondria to the appropriate location within neurons is also likely to influence their function. In this study we examined mitochondrial movement and morphology in primary cultures of rat forebrain using a mitochondrially targeted enhanced yellow fluorescent protein (mt-eYFP). Mt-eYFP-labeled mitochondria display a characteristic elongated phenotype and also move extensively. Application of glutamate to cultures results in a rapid diminution of movement and also an alteration from elongated to rounded morphology. This effect required the entry of calcium and was mediated by activation of the NMDA subtype of glutamate receptor. Treatment of cultures with an uncoupler or blocking ATP synthesis with oligomycin also stopped movement but did not alter morphology. Interestingly, application of glutamate together with the uncoupler did not prevent the changes in movement or shape but facilitated recovery after washout of the stimuli. This suggests that the critical target for calcium in this paradigm is cytosolic. These studies demonstrate that in addition to altering the bioenergetic properties of mitochondria, neurotoxins can also alter mitochondrial movement and morphology. We speculate that neurotoxin-mediated impairment of mitochondrial delivery may contribute to the injurious effects of neurotoxins.
\end{abstract}

Key words: green fluorescent protein; cytoskeleton; NMDA receptor; intracellular calcium; excitotoxicity; organelle transport

\section{Introduction}

It is widely appreciated that mitochondria in neurons are the target of a number of neurotoxins. These include agents such as rotenone, 1-methyl-4-phenyl-1,2,3,6-tetrahydropyridine (MPTP), and 3-nitroproprionic acid, and perhaps zinc, that impair mitochondrial electron transport and probably kill neurons by a combination of ATP depletion and excessive generation of reactive oxygen species (ROS) (Heales et al., 1999; Schapira, 1999; Beal, 2000; Weiss et al., 2000). Mitochondria also represent an important target in NMDA receptor-mediated excitotoxic neuronal injury. In this case, excessive calcium entry results in substantial mitochondrial calcium accumulation, mitochondrial depolarization, ROS generation, and, ultimately, cell death (Dugan et al., 1995; Reynolds and Hastings, 1995; White and Reynolds, 1995, 1996; Schinder et al., 1996; Nicholls and Budd, 2000). The neurotoxic effects of NMDA receptor activation can be occluded by prevention of mitochondrial calcium accumulation, suggesting that mitochondria are one of the critical targets of cellular calcium overload (Budd and Nicholls, 1996; Stout et al., 1998).

Studies on the role of mitochondria in neuronal injury have generally focused on the bioenergetic consequences of mitochon-

Received Feb. 24, 2003; revised June 23, 2003; accepted July 2, 2003.

These studies were supported by U.S. Army Medical Research and Materiel Command Neurotoxin Research Initiative Grant DAMD 17-98-1-8269 (I.J.R.) and Human Frontiers Science Program Grant LT0500/1999/B (J.B.B.) We thank Dr. Roger Y. Tsien for providing the mt-eYFP construct.

Correspondence should be addressed to lan J. Reynolds, Department of Pharmacology, University of Pittsburgh, W1351 Biomedical Science Tower, Pittsburgh PA 15261. E-mail: iannmda@pitt.edu.

J. B. Brocard's present address: Département de Réponse et de Dynamique Cellularies, laboratoire Canaux Ioniques et Signalisation, 38054 Grenoble Cedex, France.

Copyright $\odot 2003$ Society for Neuroscience $\quad$ 0270-6474/03/237881-08\$15.00/0 drial impairment; however, there are several important dynamics to consider that extend beyond the capacity of mitochondria to generate ATP and ROS. Mitochondria are semi-autonomous organelles that are capable of replicating their own genome, synthesizing proteins, and undergoing fission and fusion, and presumably degradation too (for review, see Scheffler, 1999). It is widely believed (although not necessarily proven) that new mitochondria are generated near the nucleus (Davis and Clayton, 1996), where they can import the large cohort of nuclear-encoded, mitochondrially targeted proteins (Scheffler, 1999). Mitochondria are then transported to appropriate cellular locations (Morris and Hollenbeck, 1993; Ligon and Steward, 2000a) that, in the case of neurons, may be a substantial distance from the presumed site of biogenesis at the cell soma. Alterations in mitochondrial distribution and morphology have been associated with various disease states. Ebneth and colleagues (1998) reported that overexpression of tau results in the failure of the cell to transport mitochondria to peripheral cell compartments, which may be of relevance to Alzheimer's disease. Alterations in mitochondrial transport and morphology have also been associated with cancer (Djaldetti, 1982), liver disease (Tandler and Hoppel, 1986), and muscular dystrophy (Nishino et al., 1998).

It is becoming clear that mitochondrial morphology is a dynamic that can be altered by a number of factors, including the induction of cell injury. Mitochondrial fragmentation has been reported in cells stimulated to undergo apoptosis, and prevention of fragmentation may be cytoprotective (Jouaville et al., 1999; Frank et al., 2001). Previous studies have also suggested calciummediated alterations in mitochondrial morphology in neurons and astrocytes (Kristal and Dubinsky, 1997; Dubinsky and Levi, 
Table 1. Summary of effects of various treatments on mitochondrial morphology and movement

\begin{tabular}{lll}
\hline & Reduction in movement & Change in morphology \\
\hline $0 \mathrm{Ca}^{2+}$ & No & No \\
Glutamate & Yes & Yes \\
Glutamate $0 \mathrm{Ca}^{2+}$ & No & No \\
$750 \mathrm{~nm}$ FCCP & Yes & No \\
$10 \mu$ M Oligomycin & Yes & No \\
Glutamate + FCCP & Yes & Yes \\
$5 \mu$ M 4-Br-A23187 & Yes & Yes \\
$100 \mu$ MMDA & Yes & Yes \\
Glutamate $+5 \mu$ M MK801 & No & No \\
$100 \mu$ Mainate & No & No
\end{tabular}

1998). In the present study we have investigated characteristics of mitochondrial movement and morphology in primary cultures of forebrain neurons transfected with mitochondrially targeted fluorescent proteins. We have found that mitochondrial depolarization inhibits movement of mitochondria in processes; however, glutamate both inhibits movement and causes a profound remodeling of mitochondria as the result of an action of calcium in the cytosol of neurons. The rate of recovery of mitochondria from this insult is promoted when mitochondrial calcium loading is prevented. These studies reveal a novel aspect of glutamatemediated injury to neurons and raise the possibility that altered mitochondrial morphology or trafficking might contribute to neuronal injury.

Some of these results have been published previously in abstract form (Rintoul et al., 2002).

\section{Materials and Methods}

DNA constructs. The mitochondrially targeted eYFP construct (generously provided by Dr. Roger Y. Tsien, University of California, San Diego) consists of the gene for eYFP, inserted into the mammalian expression vector pCDNA3 (Invitrogen). The recombinant protein is targeted to the mitochondrial matrix using the targeting sequence from subunit IV of cytochrome $c$ oxidase (Llopis et al., 1998). All other fluorescent protein expression vectors were purchased from Clontech. The cytosolic eCFP vector was constructed by excising the enhanced cyan fluorescent protein (eCFP) gene from peCFP-mito with BamHI and NotI. This fragment was then inserted into the BamHI and NotI restriction sites of the pHcRed1-N1 expression vector (replacing the HcRed gene). All plasmids were amplified using Qiagen Maxi- and Mega-prep kits according to the manufacturer's instructions.

Transfection of cortical cultures. Experiments were performed on dissociated primary cortical cultures, used between 12 and $19 \mathrm{~d}$ in vitro. Details of the culturing technique have been described previously (Kress et al., 2002). At this age in culture they are synaptically connected and vulnerable to excitotoxic cell death. Neurons were transfected using a modified calcium phosphate transfection technique (Xia et al., 1996). This typically results in transfection efficiencies of $1-2 \%$. Neurons were imaged 2-3 d after transfection.

Analysis of mitochondrial movement. Data were acquired using an acquisition system described previously (Buckman and Reynolds, 2001). Simple PCI software (Compix, Inc., Cranberry PA) was used for data acquisition. In all experiments we used a HEPES-buffered salt solution (HBSS) of the following composition (in $\mathrm{mm}$ ): $137 \mathrm{NaCl}, 5 \mathrm{KCl}, 10$ $\mathrm{NaHCO}_{3}, 20$ HEPES, 5.5 glucose, $0.6 \mathrm{KH}_{2} \mathrm{PO}_{4}, 0.6 . \mathrm{Na}_{2} \mathrm{HPO}_{4}, 1.4 \mathrm{CaCl}_{2}$, and $0.9 \mathrm{MgSO}_{4}$, adjusted to $\mathrm{pH} 7.4$ with $\mathrm{NaOH}$. In $\mathrm{Ca}^{2+}$-free experiments, $\mathrm{Ca}^{2+}$ was omitted and the buffer was supplemented with $20 \mu \mathrm{M}$ EGTA. All drugs used were purchased from Sigma (St. Louis, MO). Working solutions were prepared in HBSS from the following stock solutions: $10 \mathrm{~mm}$ glutamate in water, $10 \mathrm{~mm}$ glycine in water, $750 \mu \mathrm{M}$ carbonylcyanide-p-(trifluoromethoxy)-phenylhydrazone (FCCP) in methanol, $10 \mathrm{~mm}$ kainate in water, and $10 \mathrm{~mm}$ NMDA in water. Cells
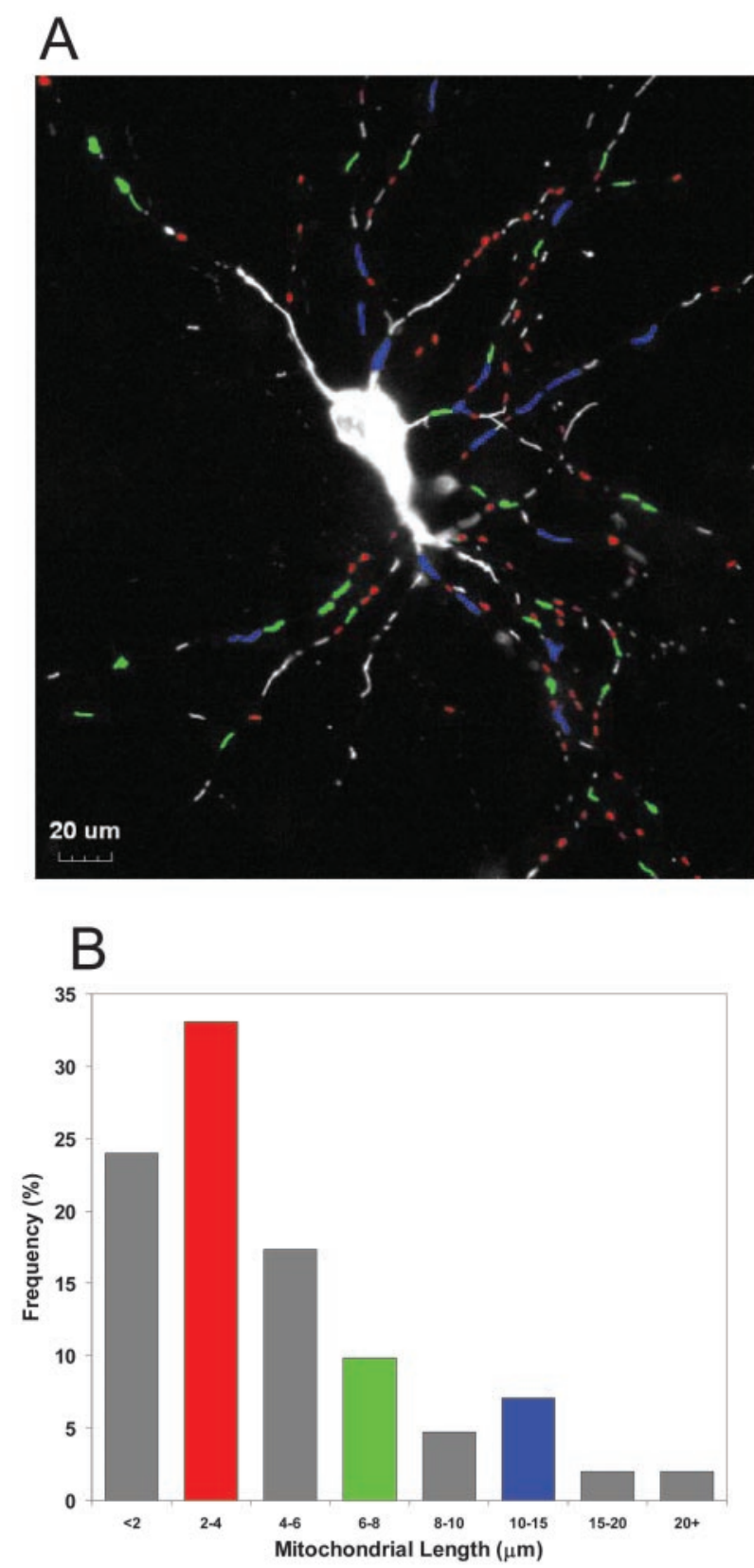

Figure 1. Identification and measurement of mitochondrial length. $A$, A fluorescence micrograph of a single cortical neuron transfected with mitochondrially targeted eYFP (grayscale image). Selected groups of mitochondria have been color coded to correspond to selected bins in the frequency histogram below. $B$, Frequency distribution of mitochondrial lengths. A representative frequency distribution derived from the above micrograph. This figure is representative of 10 additional fields in which mitochondrial length was determined.

were perfused with $5 \mathrm{ml} / \mathrm{min}$ HBSS for the duration of each experiment, and the chamber temperature was maintained at $37^{\circ} \mathrm{C}$ by heating the buffer.

Mitochondrial movement was detected by monitoring the fluorescence intensity of individual pixels in digitized images of fields containing a single transfected neuron. Twenty fluorescence digital images of a field were collected over 2 min. A $255 \times 255$ pixel subfield containing neuronal processes was selected for analysis of mitochondrial movement. Pixel intensities in successive images were subtracted. A "movement event" for each pixel was registered if the change in pixel fluorescence between successive fields exceeded 20 fluorescence units; i.e., movement was detected as an increase or decrease in individual pixel intensity over time. The threshold value of 20 fluorescence units was determined em- 

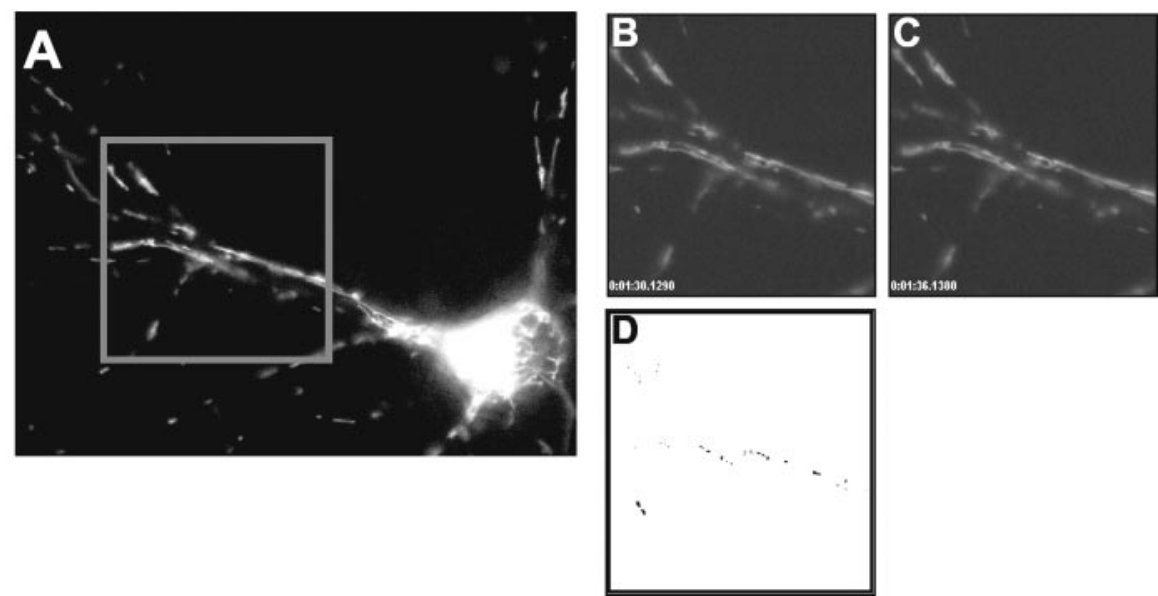

Figure 2. Measuring mitochondrial movement between two frames (see Materials and Methods). $A$, Collected image field. A $255 \times 255$ pixel subregion is selected for analysis. Individual pixel fluorescence values from successive images $(B, C)$ are subtracted. D, Threshold difference image. Dark pixels indicate "events" where change in pixel fluorescence exceeds a threshold value (20 fluorescence units). Pixel events for 20 successive images $(2 \mathrm{~min}$ ) were summated in each experiment. pirically; using a masking function in the SimplePCI software, mitochondria were found to be well defined by pixels $20 \mathrm{U}$ above background fluorescence. A quantitative measure of mitochondrial movement was obtained by summating movement events in a field over 2 min. Events were normalized by dividing the pixel events per field by the total number of pixels occupied by mitochondria. The analysis of mitochondrial movement described above was performed using custom Visual Basic macros. Determination of mitochondrial length and roundness was performed with a masking function in the SimplePCI software. The degree of mitochondrial roundness was calculated using the measured area and perimeter of identified objects and the following equation: roundness $=4 \pi$ Area $/ \mathrm{V}$ perimeter. Statistical comparisons between treatment groups were performed using Student's unpaired $t$ tests.

\section{Results}

Mitochondrial size and movement

In this study we have made use of mt-eYFP
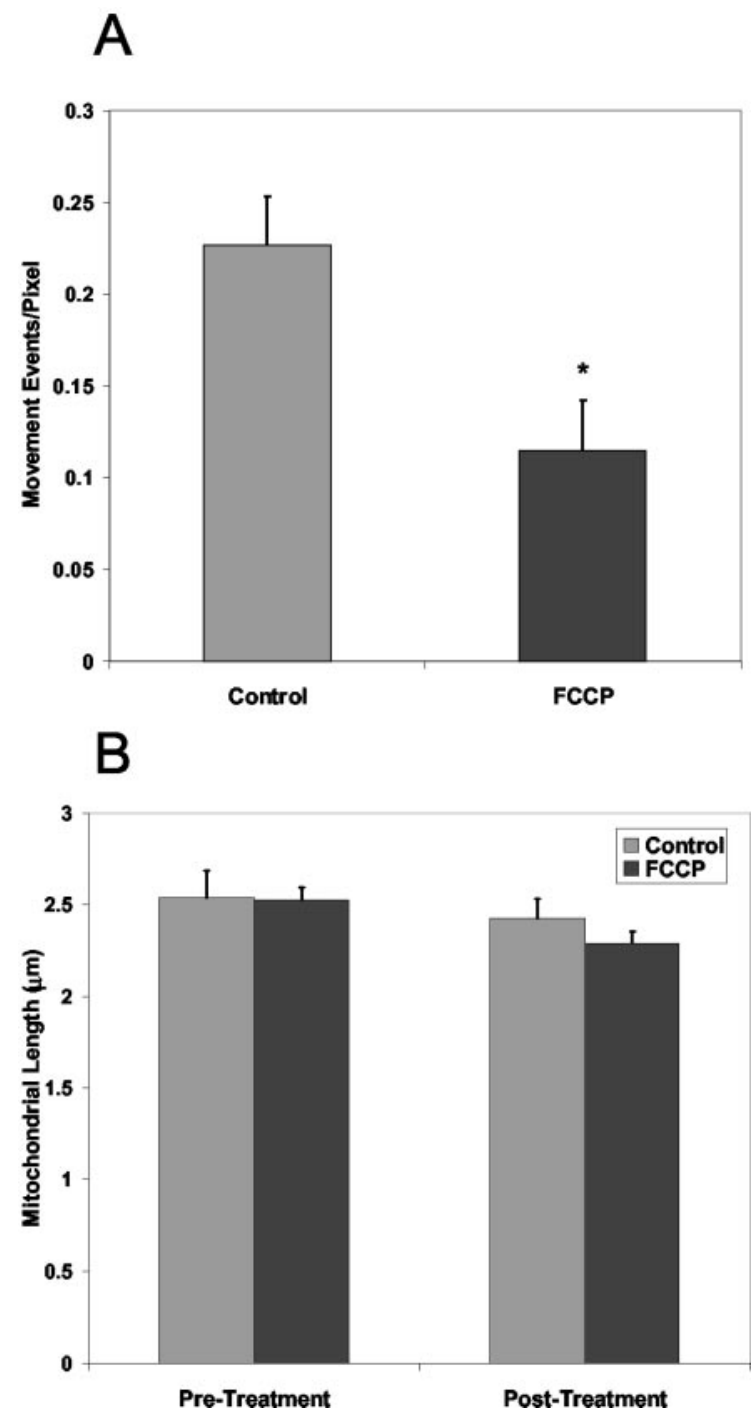

Figure 3. Effect of $\mathrm{FCCP}$ on mitochondrial movement and morphology. $A$, Mean movement events per pixel measured after treatment with $750 \mathrm{~nm} F C C P$. $B$, Mean mitochondrial length after treatment of transfected cells with $750 \mathrm{~nm} \mathrm{FCCP.}{ }^{*}$ Significantly different from control $(p<$ $0.05 ; t$ test; control, $n=10 ; \mathrm{FCCP}, n=7)$. to label neuronal mitochondria. Using calcium phosphate we routinely obtained 1-2\% transfection efficiency. With this efficiency the density of transfected cells was such that typically a single labeled cell was present per field, although sometimes additional labeled processes traversed the field. The imaging conditions were optimized to record individual mitochondria in processes, with the result that cell bodies were usually overexposed. The limited extent of labeling provided relatively simple images that were amenable to semi-automated image analysis. Thus, we were able to determine the characteristics of mitochondrial length quite readily (Fig. 1). Size varied considerably, but the numerical majority of objects was relatively small. This probably reflects the presence of bright, small mitochondria as well as marginally labeled organelles with fluorescence intensity that did not exceed threshold for the entire length of the organelle. The preponderance of small objects in these images diminishes the impact of the modest number of long mitochondria on the mean length, although the latter objects tend to stand out on visual examination of the images.

We also used semi-automated approaches to estimate movement (Fig. 2). To determine the overall movement in a field, we movies and then counted events that were defined as bright-todark or dark-to-bright transitions of individual pixels that exceeded an arbitrarily defined threshold. These events were summed over 2 min of data acquisition (20 frames). Typically, this approach identified 5,000-20,000 events per 2 min period. In cells that had been fixed in paraformaldehyde before imaging, the number of events detected in the same time frame was typically $<20$, indicating that the noise associated with the recording system did not make a meaningful contribution to this signal. This method detects both directed movement of objects as well as lateral movement of otherwise stationary objects ("wiggling"), although the latter type of movement was quite limited in mitochondria constrained within processes. We also tracked individual objects using the object tracking module of Simple PCI to determine the velocity of the most rapidly moving objects within fields. We visually identified individual objects from eight different fields of cells on the basis of their rapid movement and estimated a mean velocity of $0.63 \pm 0.09 \mathrm{~m} / \mathrm{sec}$. These rapidly moving mitochondria were inevitably small in length. used an approach that involves subtraction of adjacent images of 


\section{Stimulus-induced alterations in size and movement}

We next examined the effects of the mitochondrial uncoupler FCCP, which rapidly dissipates the mitochondrial membrane potential. This can deplete cellular ATP both by preventing its synthesis and also by consumption of ATP by the $\mathrm{F}_{1} \mathrm{~F}_{\mathrm{O}}$ ATPase. A $5 \mathrm{~min}$ application of FCCP promptly decreased the movement of mitochondria without altering mitochondrial size (Fig. 3). This suggests that movement requires an adequate ATP supply, an intact mitochondrial membrane potential, or possibly both; however, treatment with $10 \mu \mathrm{M}$ oligomycin produced a similar attenuation of mitochondrial movement (Fig. 4C). This suggests that local depletion of ATP and not changes in membrane potential may impede the movement of mitochondria.

Exposure of neurons to $30 \mu \mathrm{M}$ glutamate with $1 \mu \mathrm{M}$ glycine produced a somewhat different response. Although the mitochondria clearly stopped moving within 2-4 min of glutamate application, we also noted a profound alteration in mitochondrial morphology over the same time frame (Fig. 4). Thus, mitochondria that were initially thread-like in structure became notably rounded. It is also possible that there was fragmentation, such that individual thread-like objects became more than one rounded object; however, it is difficult to conclusively establish that fragmentation explicitly occurs rather than there being adjacent or overlapping objects that become distinguishable when rounded. Although fragmentation may occur, it is not clear that it would account for the majority of the morphological changes evident in these images. Quantitation of the alteration in both movement and length revealed that glutamate effectively decreased mitochondrial movement and also produced a significant decrease in mitochondrial length (Fig. 4). Sequential analysis of length and object roundness during glutamate exposure indicated that the decrease in length was associated with a simultaneous increase in roundness.

\section{Mechanisms underlying glutamate-induced morphological alteration}

An evaluation of the mechanisms underlying this effect of glutamate suggested a critical contribution of NMDA receptors. Applying glutamate in the presence of MK801 $(5 \mu \mathrm{M})$ completely blocked the effects of the agonist, whereas the application of $\operatorname{NMDA}(100 \mu \mathrm{M}$ with $1 \mu \mathrm{M}$ glycine) also decreased mitochondrial length and diminished movement. Interestingly, kainate (100 $\mu \mathrm{M})$ did not mimic the effects of glutamate. Given that kainate effectively depolarizes the plasma membrane but results in a much smaller calcium load (Stout and Reynolds, 1999), this ob7; Glutamate, $n=10)$.
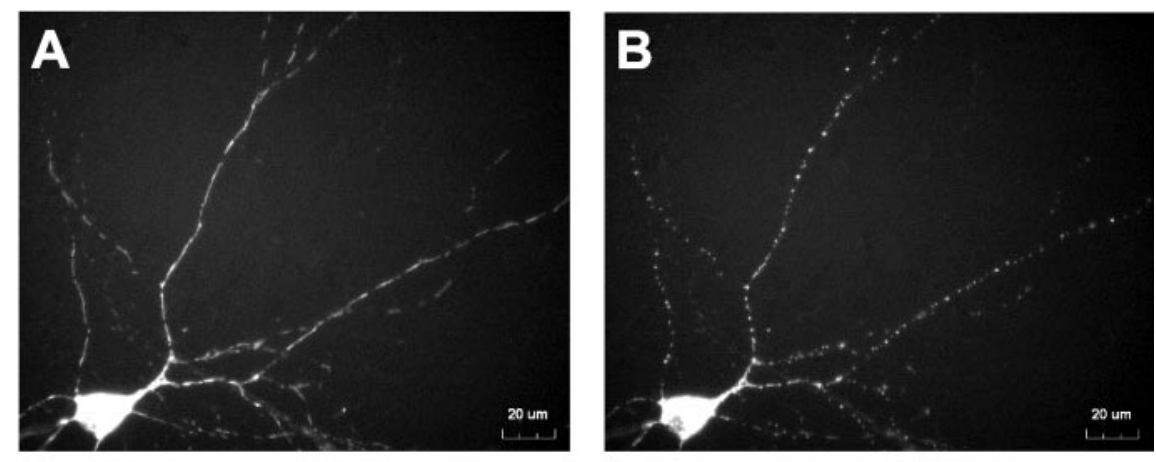

D
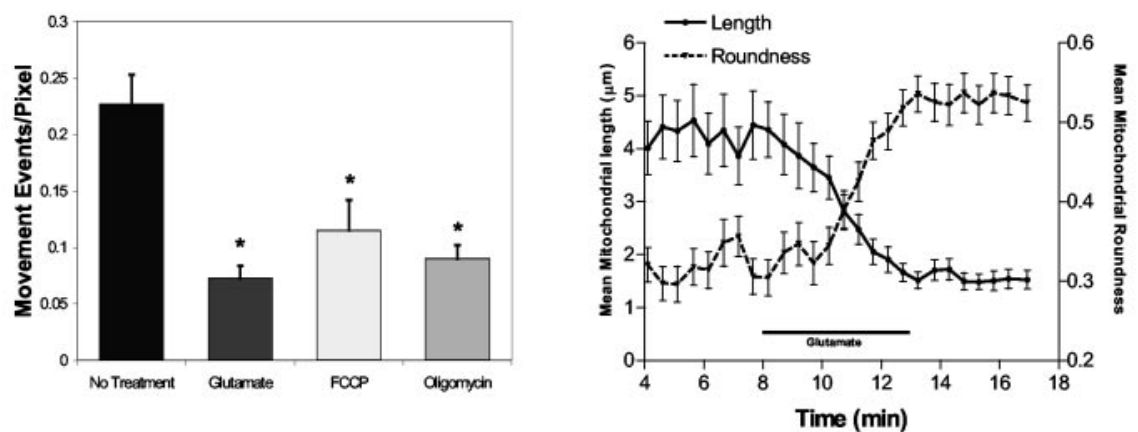

$\mathbf{F}$

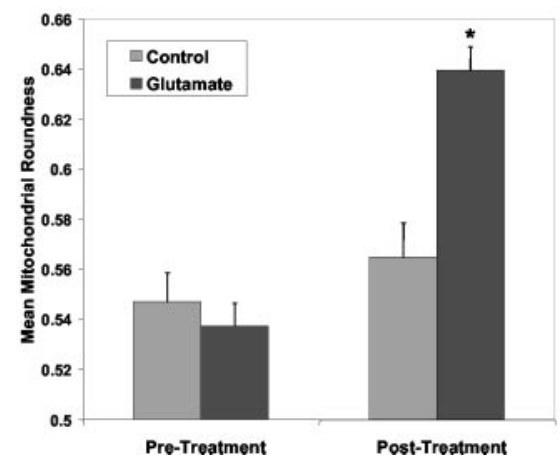

Figure 4. Glutamate induction of mitochondrial remodeling in cortical cultures. Shown are representative micrographs of cortical neurons transfected with mt-eYFP, before $(A)$ and after $(B)$ a 5 min treatment with $30 \mu \mathrm{m}$ glutamate/1 $\mu \mathrm{m}$ glycine. $C$, Mean measured mitochondrial movement after treatment of transfected neurons with $30 \mu \mathrm{m}$ glutamate/ $1 \mu \mathrm{m}$ glycine. Reductions in movement after FCCP and oligomycin treatments are show for comparison. *Significantly different from control ( $p<$ 0.05; $t$ test; No Treatment, $n=7$; Glutamate, $n=5 ; \mathrm{FCCP}, n=7$; Oligomycin, $n=7)$. D, Representative traces of mean mitochondrial length and roundness after treatment with glutamate/glycine, derived from the image sequence shown in $A$ and $B$. Similar results were obtained in five additional fields of cells. $E$, Pooled data of mitochondrial length measured after treatment with glutamate/glycine. * Significantly different from control ( $p<0.05 ; t$ test; Control, $n=7$; Glutamate, $n=10)$. F, Mean mitochondrial roundness after glutamate/glycine treatment. * Significantly different from control $(p<0.05 ; t$ test; Control, $n=$

servation suggested a key role for a substantial intracellular calcium accumulation. Supporting this hypothesis, treatment of cultures with the calcium ionophore 4-Br-A23187 (5 $\mu \mathrm{M})$ produced the same effects as glutamate on mitochondrial motility and morphology. Applying glutamate in a calcium-free buffer also completely blocked the effect, indicating that the remodeling was the consequence of NMDA receptor-mediated calcium entry and presumably independent of either plasma membrane depolarization or intracellular sodium accumulation.

We have shown previously that FCCP can protect neurons from glutamate excitotoxicity, ostensibly as the result of preventing mitochondrial calcium accumulation. Interestingly, applying 

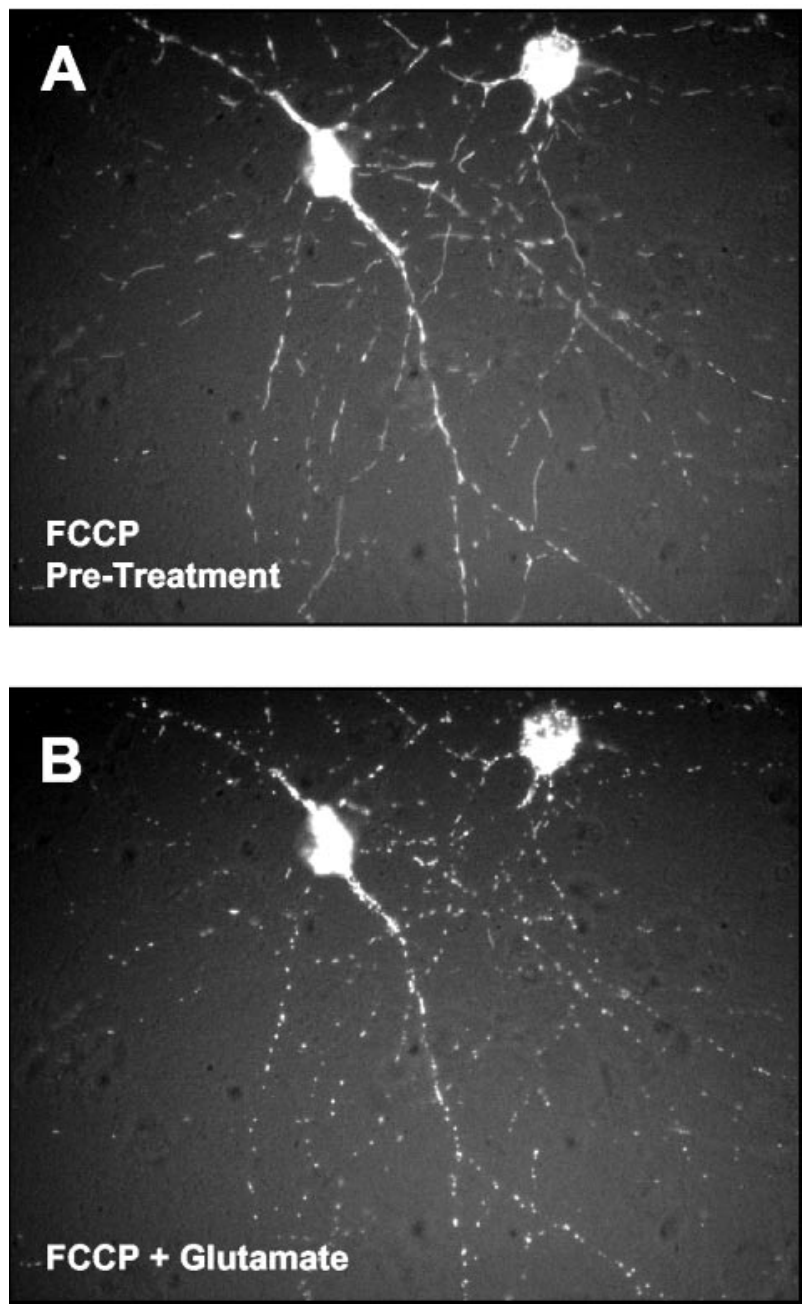

Figure 5. Glutamate-induced mitochondrial remodeling does not require mitochondrial calcium uptake. Shown are representative micrographs of a neuron pretreated $(A)$ for 3 min with $750 \mathrm{~nm}$ FCCP and then superfused ( $B$ ) with $30 \mu \mathrm{m}$ glutamate/1 $\mu \mathrm{m}$ glycine in the continued presence of $750 \mathrm{~nm}$ FCCP.

glutamate in the presence of FCCP had no impact on the calciumdependent remodeling of mitochondrial shape (Fig. 5). Under these conditions there is unlikely to be a substantial accumulation of calcium in the mitochondrial matrix, so this finding indicates that the probable locus of action of calcium in this experiment is in the cytosol.

\section{Is the mitochondrial remodeling a consequence of dendrite remodeling?}

Previous studies have reported a prompt and reversible alteration in the structure of the processes of cultured neurons in response to NMDA receptor activation (Park et al., 1996) that shows a similar time course to the phenomenon observed here. To evaluate the structure of the dendrites in comparison with the mitochondria, we cotransfected cells with a cytosolic form of CFP along with mt-eYFP. A parallel analysis of the two markers revealed that the mitochondrial remodeling was accompanied by beading of dendrites, so that the structural remodeling appears to occur concomitantly (Fig. 6); however, mitochondria do not appear to be specifically associated with either the swollen varicosities or the narrowed parts of the process. This suggests that it is unlikely that the remodeling is the consequence of the mitochondria essentially being "pinched off" by the alteration in the structure of the dendrite. It is also apparent that although the mor- phology of the dendrites is clearly altered by glutamate treatment, they remain contiguous rather than fragmented. This indicates that the rounded mitochondria do not simply represent cellular debris at the end of a gross injury process. This conclusion is reinforced by the studies on the recovery of normal morphology described in the next section.

\section{Mitochondrial shape recovers after glutamate exposure}

We next sought to determine whether mitochondrial shape recovered after glutamate exposure. We assessed mitochondrial size 1 and $2 \mathrm{hr}$ after glutamate treatment. By $1 \mathrm{hr}$ into the recovery period, the glutamate-treated neurons were still clearly different from controls in that movement was limited and the rounded mitochondria presented as the dominant phenotype. Interestingly, however, neurons exposed to glutamate in the presence of FCCP showed a much greater degree of recovery of morphology at this time point (Fig. 7) and were indistinguishable from controls. At longer time points ( $2 \mathrm{hr}$ ) but before frank injury was apparent, both populations of treated cells had recovered to control values. These data suggest that the FCCP co-treatment facilitated what might be considered a normal recovery process.

\section{Discussion}

The main findings of this study are that glutamate, as a consequence of NMDA receptor activation, produces a rapid and substantial remodeling of mitochondrial morphology and also causes a cessation of mitochondrial movement. These previously unappreciated aspects of the action of glutamate at concentrations just sufficient to cause excitotoxic injury suggest that there may be effects of glutamate on mitochondria that extend beyond the bioenergetic and ROS generation effects that we and other laboratories have documented previously. In particular, these findings suggest that neuronal injury might alter the trafficking of mitochondria to cellular destinations where ATP synthesis is required and thus impair cellular function.

There is an emerging appreciation that mitochondrial morphology is a dynamic parameter that may be associated with cell injury. In several different models of apoptosis it has been reported that the normal mitochondrial reticulum becomes fragmented after the application of apoptotic stimuli (Desagher and Martinou, 2000; Frank et al., 2001). Fragmentation of mitochondria may be a consequence of the association of proteins such as dynamin-related protein 1 (DRP1) with mitochondria. Overexpression of this protein causes fragmentation (Frank et al., 2001; Filiano et al., 2002), whereas a dominant-negative form of DRP1 decreases sensitivity to mitochondrially mediated apoptosis (Frank et al., 2001). It is not clear whether such mechanisms are activated by calcium, or even whether DRP1 is normally present in neurons. A different family of proteins appears to mediate fusion of mitochondria (Santel and Fuller, 2001). It is also possible that an inhibition of an ongoing fusion reaction could result in an apparent fragmentation or rounding, although there is no precedent for this kind of effect as far as we are aware.

Alterations in mitochondrial morphology in both neurons and astrocytes in response to calcium loading has also been reported by Dubinsky and colleagues (Kristal and Dubinsky, 1997; Dubinsky and Levi, 1998), who also found that mitochondria changed from a rod-like to spherical morphology when challenged. In these studies the authors concluded that the shape change was caused by mitochondrial permeability transition. Our preliminary experiments indicated that the classic transition 
inhibitor cyclosporin A was ineffective in preventing these morphological changes (data not shown). There is also the ambiguity in these studies that the shape change was detected with a membrane potentialsensitive dye under conditions in which the membrane potential is clearly a variable (Schinder et al., 1996; White and Reynolds, 1996; Duchen et al., 1998). Nevertheless, our findings clearly support the morphological conclusions in these earlier reports. The consequence of the alteration in morphology for mitochondrial function is much less clear. It has been proposed that the elongated or reticulated form of mitochondria is beneficial on the basis of studies in cardiac myocytes (Amchenkova et al., 1988). Thus, because of the cable properties of mitochondria, the proton motive force generated by the electron transport chain can be effectively distributed across the reticulum and thus facilitate ATP synthesis. This advantage is hypothetical at this point, however, because it remains unclear whether the ability of mitochondria to generate a proton motive force is spatially limited. Because both FCCP or an excessive calcium load result in mitochondrial depolarization in our experiments, it is difficult to assess mitochondrial function in mitochondria after rounding, so we cannot readily establish whether function is modified as a direct consequence of the shape change.

A second dramatic consequence of the action of glutamate is the cessation of mitochondrial movement. The directional movement of mitochondria in neurons is accomplished using the microtubule network and can be inhibited by stabilizing microtubules with drugs like nocodazole (Morris and Hollenbeck, 1995; Overly et al., 1996; Ligon and Steward, 2000b). Movement on the actin cytoskeleton has also been reported (Morris and Hollenbeck, 1995). Movement is presumably driven by motor proteins such as kinesin and dynein (Goldstein, 2001) as well as myosin (Morris and Hollenbeck, 1995), although the specific interactions governing this mechanism in the context of neuronal mitochondria have not been clearly established. The cytoskeleton is clearly subject to profound alterations in the face of a robust calcium load. The actin cytoskeleton can be modified by calcium-mediated activation of gelsolin, whereas calcineurin activation results in dephosphorylation of microtubule-associated proteins (van Rossum and Hanisch, 1999). Fodrin is a substrate for calpains, which may also be activated under these circumstances (van Rossum and Hanisch, 1999), although we have found that calpain inhibitors do not prevent this effect of glutamate (data not shown). Thus, there are many factors that could alter the substrates on which mitochondria move. Movement is also an ATP-dependent process, and the depolarization of mitochondria combined with the potential impairment of function associated with the calcium load could deplete local ATP concentrations quite rapidly. Thus, a number of different mechanisms could contribute to the cessation of movement. The mechanisms responsible for the alteration in movement may also underlie

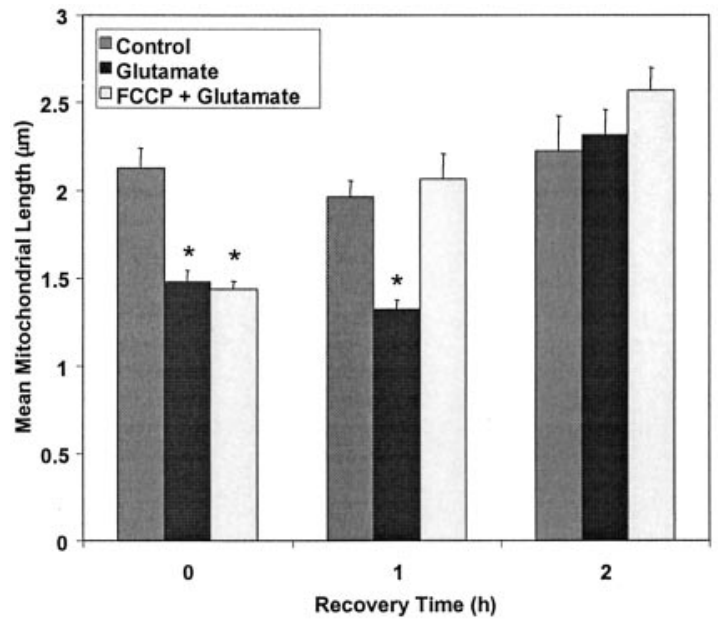

Figure 7. Recovery of mitochondria after glutamate and combined FCCP + glutamate treatments. Mitochondrial length was measured immediately after treatment of transfected cells with 30 $\mu \mathrm{m}$ glutamate/ $1 \mu \mathrm{m}$ glycine and subsequently measured 1 and $2 \mathrm{hr}$ after treatment. * Significantly different from control $(p<0.05 ; t$ test; $n$ ranges between 7 and 15 for each bar). 
the shape change. If the rod-like structure associated with normal mitochondria is essentially a function of the organelle being stretched out on a cytoskeletal structure, the dissolution of that structure could result in the rounding that we observe.

It is interesting to consider the role that these glutamatemediated effects on mitochondria might have in the expression of excitotoxic injury. The effects are clearly produced by concentrations and exposure times of glutamate that are around the threshold for neuronal injury; however, the changes in shape and movement that we observe are the same in the presence and absence of FCCP. This is notable, because we have demonstrated previously that the combined application of FCCP with glutamate protects neurons from excitotoxic injury, ostensibly as the result of preventing mitochondrial calcium accumulation (Stout et al., 1998). Because both rounding and movement cessation occur in the presence of FCCP with glutamate, one can conclude that rounding of mitochondria or their temporary immobilization does not inevitably lead to injury; however, it is interesting to note that the presence of FCCP during glutamate exposure clearly facilitates the recovery of the normal morphological phenotype. This suggests that the protection of mitochondrial function (by preventing calcium overload) aids in the restoration of mitochondria to their normal state. Thus, in this experimental paradigm, the alteration of mitochondrial morphology would be considered a symptom rather than a cause of cell injury. Indeed, this raises the possibility that either the shape or movement change could be an attempt to protect the cell from injury, although what exactly is accomplished by this maneuver remains to be established.

More broadly, these experiments clearly establish an unappreciated dynamic that exists between the distribution of mitochondria within cells and their bioenergetic status. Under conditions of less acute injury mechanisms, one might anticipate that impaired mitochondrial function would alter the ability of mitochondria to move and thereby prevent the normal distribution of mitochondria within neurons. Under conditions of chronic stress, this could stop new mitochondria from being delivered to distal parts of the neuron or possibly even prevent the retrieval of dysfunctional mitochondria to the cell body for degradation, either of which may result in cell damage. When one considers the number of endogenous toxins (such as zinc, nitric oxide, and oxidative stress) as well as xenobiotics (such as MPTP, rotenone, 3-nitroproprionic acid), that impair mitochondrial function and dissipate the mitochondrial membrane potential, it seems likely that the alteration of the trafficking and morphology of mitochondria is likely to be a broadly important phenomenon associated with neuronal injury.

\section{References}

Amchenkova AA, Bakeeva LE, Chentsov YS, Skulachev VP, Zorov DB (1988) Coupling membranes as energy-transmitting cables. I. Filamentous mitochondria in fibroblasts and mitochondrial clusters in cardiomyocytes. J Cell Biol 107:481-492.

Beal MF (2000) Energetics in the pathogenesis of neurodegenerative diseases. Trends Neurosci 23:298-304.

Buckman JF, Reynolds IJ (2001) Spontaneous changes in mitochondrial membrane potential in cultured neurons. J Neurosci 21:5054-5065.

Budd SL, Nicholls DG (1996) Mitochondria, calcium regulation and acute glutamate excitotoxicity in cultured cerebellar granule cells. J Neurochem 67:2282-2291.

Davis AF, Clayton DA (1996) In situ localization of mitochondrial DNA replication in intact mammalian cells. J Cell Biol 135:883-893.

Desagher S, Martinou JC (2000) Mitochondria as the central control point of apoptosis. Trends Cell Biol 10:369-377.
Djaldetti M (1982) Mitochondrial abnormalities in the cells of myeloma patients. Acta Haematologica 68:241-248.

Dubinsky JM, Levi Y (1998) Calcium induced activation of the mitochondrial permeability transition in hippocampal neurons. J Neurosci Res 53:728-741.

Duchen MR, Leyssens A, Crompton M (1998) Transient mitochondrial depolarizations reflect focal sarcoplasmic reticular calcium release in single rat cardiomyocytes. J Cell Biol 142:975-988.

Dugan LL, Sensi SL, Canzoniero LMT, Handran SD, Rothman SM, Lin T-S, Goldberg MP, Choi DW (1995) Mitochondrial production of reactive oxygen species in cortical neurons after exposure to NMDA. J Neurosci 15:6377-6388.

Ebneth A, Godemann R, Stamer K, Illenberger S, Trinczek B, Mandelkow E (1998) Overexpression of tau protein inhibits kinesin-dependent trafficking of vesicles, mitochondria, and endoplasmic reticulum: implications for Alzheimer's disease. J Cell Biol 143:777-794.

Filiano AJ, Rintoul GL, Brocard JB, Votyakova TV, Reynolds IJ (2002) Mitochondrial homeostasis and neuronal injury in primary cortical cultures. Soc Neurosci Abstr 32:297.2.

Frank S, Gaume B, Bergmann-Leitner ES, Leitner WW, Robert EG, Catez F, Smith CL, Youle RJ (2001) The role of dynamin-related protein 1, a mediator of mitochondrial fission, in apoptosis. Dev Cell 1:515-525.

Goldstein LS (2001) Molecular motors: from one motor many tails to one motor many tales. Trends Cell Biol 11:477-482.

Heales SJ, Bolaños JP, Stewart VC, Brookes PS, Land JM, Clark JB (1999) Nitric oxide, mitochondria and neurological disease. Biochim Biophys Acta 1410:215-228.

Jouaville LS, Pinton P, Bastianutto C, Rutter GA, Rizzuto R (1999) Regulation of mitochondrial ATP synthesis by calcium: evidence for long term metabolic priming. Proc Natl Acad Sci USA 96:13807-13812.

Kress GJ, Dineley KE, Reynolds IJ (2002) The relationship between intracellular free iron and cell injury in cultured neurons, astrocytes, and oligodendrocytes. J Neurosci 22:5848-5855.

Kristal BS, Dubinsky JM (1997) Mitochondrial permeability transition in the central nervous system: induction by calcium cycling-dependent and independent pathways. J Neurochem 69:524-538.

Ligon LA, Steward O (2000a) Movement of mitochondria in the axons and dendrites of cultured hippocampal neurons. J Comp Neurol 347:340-350.

Ligon LA, Steward O (2000b) Role of microtubules and actin filaments in the movement of mitochondria in the axons and dendrites of cultured hippocampal neurons. J Comp Neurol 427:351-361.

Llopis J, McCaffery JM, Miyawaki A, Farquhar MG, Tsien RY (1998) Measurement of cytosolic, mitochondrial and Golgi pH in single living cells with green fluorescent proteins. Proc Natl Acad Sci USA 95:6803-6808.

Morris RL, Hollenbeck PJ (1993) The regulation of bidirectional mitochondrial transport is coordinated with axonal outgrowth. J Cell Sci 104:917-927.

Morris RL, Hollenbeck PJ (1995) Axonal transport of mitochondria along microtubules and F-actin in living vertebrate neurons. J Cell Biol 131:1315-1326.

Nicholls DG, Budd SL (2000) Mitochondria and neuronal survival. Physiol Rev 80:315-360.

Nishino I, Kobayashi O, Goto Y, Kurihara M, Kumagai K, Fujita T, Hashimoto K, Horai S, Nonaka I (1998) A new congenital muscular dystrophy with mitochondrial structural abnormalities. Muscle Nerve 21:40-47.

Overly CC, Rieff HI, Hollenbeck PJ (1996) Organelle motility and metabolism in axons vs dendrites of cultured hippocampal neurons. J Cell Sci 109:971-980.

Park JS, Bateman MC, Goldberg MP (1996) Rapid alterations in dendrite morphology during sublethal hypoxia or glutamate receptor activation. Neurobiol Dis 3:215-227.

Reynolds IJ, Hastings TG (1995) Glutamate induces the production of reactive oxygen species in cultured forebrain neurons following NMDA receptor activation. J Neurosci 15:3318-3327.

Rintoul GL, Filiano AJ, Brocard JB, Werner D, Reynolds IJ (2002) Mitochondrial remodeling in response to excitotoxic glutamate treatment of primary cortical cultures. Soc Neurosci Abstr 32:297.1.

Santel A, Fuller MT (2001) Control of mitochondrial morphology by a human mitofusin. J Cell Sci 114:867-874.

Schapira AH (1999) Mitochondrial involvement in Parkinson's disease, 
Huntington's disease, hereditary spastic paraplegia and Friedreich's ataxia. Biochim Biophys Acta 1410:159-170.

Scheffler IE (1999) Mitochondria. New York: Wiley.

Schinder AF, Olson EC, Spitzer NC, Montal M (1996) Mitochondrial dysfunction is a primary event in glutamate neurotoxicity. J Neurosci 16:6125-6133.

Stout AK, Reynolds IJ (1999) High-affinity calcium indicators underestimate increases in intracellular calcium concentrations associated with excitotoxic glutamate stimulations. Neuroscience 89:91-100.

Stout AK, Raphael HM, Kanterewicz BI, Klann E, Reynolds IJ (1998) Glutamate-induced neuron death requires mitochondrial calcium uptake. Nat Neurosci 1:366-373.

Tandler B, Hoppel CL (1986) Studies on giant mitochondria. Ann NY Acad Sci 488:65-81. van Rossum D, Hanisch UK (1999) Cytoskeletal dynamics in dendritic spines: direct modulation by glutamate receptors? Trends Neurosci 22:290-295.

Weiss JH, Sensi SL, Koh JY (2000) Zn(2+): a novel ionic mediator of neural injury in brain disease. Trends Pharmacol Sci 21:395-401.

White RJ, Reynolds IJ (1995) Mitochondria and $\mathrm{Na}^{+} / \mathrm{Ca}^{2+}$ exchange buffer glutamate-induced calcium loads in cultured cortical neurons. J Neurosci 15:1318-1328.

White RJ, Reynolds IJ (1996) Mitochondrial depolarization in glutamatestimulated neurons: an early signal specific to excitotoxin exposure. J Neurosci 16:5688-5697.

Xia Z, Dudek H, Miranti CK, Greenberg ME (1996) Calcium influx via the NMDA receptor induces immediate early gene transcription by a MAP kinase/ERK-dependent mechanism. J Neurosci 16:5425-5436. 